\title{
GENERIC CHARACTER SHEAVES ON DISCONNECTED GROUPS AND CHARACTER VALUES
}

\author{
G. LUSZTIG
}

\begin{abstract}
We relate a generic character sheaf on a disconnected reductive group with a character of the rational points of the group over a finite field extending a known result in the connected case.
\end{abstract}

\section{INTRODUCTION}

The theory of character sheaves L3] on a reductive group $G$ over an algebraically closed field and the theory of irreducible characters of $G$ over a finite field are two parallel theories; the first one is geometric (involving intersection cohomology complexes on $G$ ), and the second one involves functions on the group of rational points of $G$. In the case where $G$ is connected, a bridge between the two theories was constructed in [L1] and strengthened in [L2], [S]. In this paper we begin the construction of the analogous bridge in the general case, extending the method of [L1]. Here we restrict ourselves to character sheaves which are "generic" (in particular, their support is a full connected component of $G$ ) and show how such character sheaves are related to characters of representations (see Theorem 1.2). The same problem for certain non-generic character sheaves in the case where $G$ is $G L_{n}$ extended by an outer automorphism and the characteristic is odd is considered in $[\mathrm{W}$.

\section{Contents}

1. Statement of the Theorem.

2. Constructing representations of $G^{F}$.

3. Proof of Theorem 1.2.

\section{Statement of the Theorem}

1.1. Let $\mathbf{k}$ be an algebraic closure of a finite field $\mathbf{F}_{q}$. Let $G$ be a reductive algebraic group over k with identity component $G^{0}$ such that $G / G^{0}$ is cyclic, generated by a fixed connected component $D$. We assume that $G$ has a fixed $\mathbf{F}_{q}$-rational structure with Frobenius map $F: G \rightarrow G$ such that $F(D)=D$. Let $l$ be a prime number invertible in $\mathbf{k}$; let $\overline{\mathbf{Q}}_{l}$ be an algebraic closure of the $l$-adic numbers. All group representations are assumed to be finite dimensional over $\overline{\mathbf{Q}}_{l}$. We say "local system" instead of " $\overline{\mathbf{Q}}_{l}$-local system".

Received by the editors April 7, 2007.

2000 Mathematics Subject Classification. Primary 20G99.

Supported in part by the National Science Foundation.

(C)2008 American Mathematical Society Reverts to public domain 28 years from publication 
Let $\mathcal{B}$ be the variety of Borel subgroups of $G^{0}$. Now $F: G \rightarrow G$ induces a morphism $\mathcal{B} \rightarrow \mathcal{B}$ denoted again by $F$. We fix $B^{*} \in \mathcal{B}$ and a maximal torus $T$ of $B^{*}$ such that $F\left(B^{*}\right)=B^{*}, F(T)=T$. Let $U^{*}$ be the unipotent radical of $B^{*}$. Let $N B^{*}$ (resp. $\left.N T\right)$ be the normalizer of $B^{*}$ (resp. $T$ ) in $G$. Let $\tilde{T}=N T \cap N B^{*}$, a closed $F$-stable subgroup of $G$ with identity component $T$. Let $\tilde{T}_{D}=\tilde{T} \cap D$.

Let $\mathcal{N}=N T \cap G^{0}$. Let $W=\mathcal{N} / T$ be the Weyl group. Let $\underline{D}: T \stackrel{\sim}{\rightarrow} T$, $\underline{D}: W \stackrel{\sim}{\rightarrow} W$ be the automorphisms induced by $\operatorname{Ad}(d): \mathcal{N} \rightarrow \mathcal{N}$, where $d$ is any element of $\tilde{T}_{D}$. Now $F: \mathcal{N} \rightarrow \mathcal{N}$ induces an automorphism of $W$ denoted again by $F$. For $w \in W$ let $[w]$ be the inverse image of $w$ under the obvious map $\mathcal{N} \rightarrow W$ and let $\underline{w}$ be the automorphism $\operatorname{Ad}(x): T \rightarrow T$ for any $x \in[w]$. For $w \in W$ let $\mathcal{O}_{w}$ be the $G^{0}$-orbit in $\mathcal{B} \times \mathcal{B}\left(G^{0}\right.$ acting by simultaneous conjugation on both factors) that contains $\left(B^{*}, x B^{*} x^{-1}\right)$ for some/any $x \in[w]$. Define the "length function" $l: W \rightarrow \mathbf{N}$ by $l(w)=\operatorname{dim} \mathcal{O}_{w}-\operatorname{dim} \mathcal{B}$. For any $y \in G^{0}$ we define $k(y) \in \mathcal{N}$ by $y \in U^{*} k(y) U^{*}$. For $y \in G^{0}, \tau \in \tilde{T}$ we have $k\left(\tau y \tau^{-1}\right)=\tau k(y) \tau^{-1}$ and $F(k(y))=k(F(y))$. For $x \in G^{0}$ we define $F_{x}: G \rightarrow G$ by $F_{x}(g)=x F(g) x^{-1}$; this is the Frobenius map for an $\mathbf{F}_{q}$-rational structure on $G$. (Indeed if $y \in G^{0}$ is such that $x=y^{-1} F(y)$, then $\operatorname{Ad}(y): G \stackrel{\sim}{\rightarrow} G$ carries $F_{x}$ to $F$.) If $w \in W$ satisfies $\underline{D}(w)=w$ and $x \in[w]$, then $T, \tilde{T}$ are $F_{x}$-stable; thus $F_{x}$ is the Frobenius map for an $\mathbf{F}_{q}$-rational structure on $\tilde{T}$ whose group of rational points is $\tilde{T}^{F_{x}}$. Since $\tilde{T}_{D}^{F_{x}}$ is the set of rational points of $\tilde{T}_{D}$ (a homogeneous $T$-space under left translation) for the rational structure defined by $F_{x}: \tilde{T}_{D} \rightarrow \tilde{T}_{D}$, we have $\tilde{T}_{D}^{F_{x}} \neq \emptyset$.

Let $Z_{\emptyset}=\left\{\left(B_{0}, g\right) \in \mathcal{B} \times D ; g B_{0} g^{-1}=B_{0}\right\}$. Let $d \in \tilde{T}_{D}$. We set

$$
\dot{Z}_{\emptyset, d}=\left\{\left(h_{0} U^{*}, g\right) \in\left(G^{0} / U^{*}\right) \times D ; h_{0}^{-1} g h_{0} d^{-1} \in B^{*}\right\} .
$$

Define $a_{\emptyset}: \dot{Z}_{\emptyset, d} \rightarrow Z_{\emptyset}$ by $\left(h_{0} U^{*}, g\right) \mapsto\left(h_{0} B^{*} h_{0}^{-1}, g\right)$. Now $a_{\emptyset}$ is a principal $T$ bundle, where $T$ acts (freely) on $\dot{Z}_{\emptyset, d}$ by $t_{0}:\left(h_{0} U^{*}, g\right) \mapsto\left(h_{0} t_{0}^{-1}, g\right)$. Define $p_{\emptyset}$ : $Z_{\emptyset} \rightarrow D$ by $\left(B_{0}, g\right) \mapsto g$. We define $b_{\emptyset}: \dot{Z}_{\emptyset, d} \rightarrow T$ by $\left(h_{0} U^{*}, g\right) \mapsto k\left(h_{0}^{-1} g h_{0} d^{-1}\right)$. Note that $b_{\emptyset}$ commutes with the $T$-actions where $T$ acts on $T$ by

(a) $t_{0}: t \mapsto t_{0} t \underline{D}\left(t_{0}^{-1}\right)$.

Let $\mathcal{L}$ be a local system of rank 1 on $T$ such that

(i) $\mathcal{L}^{\otimes n} \cong \overline{\mathbf{Q}}_{l}$ for some $n \geq 1$ invertible in $\mathbf{k}$;

(ii) $\underline{D}^{*} \mathcal{L} \cong \mathcal{L}$.

From (i), (ii) we see (using [L3, 28.2(a)]) that $\mathcal{L}$ is equivariant for the $T$-action (a) on $T$. Hence $b_{\emptyset}^{*} \mathcal{L}$ is a $T$-equivariant local system on $\dot{Z}_{\emptyset, d}$. Since $a_{\emptyset}$ is a principal $T$-bundle there is a well-defined local system $\tilde{\mathcal{L}}_{\emptyset}$ on $Z_{\emptyset}$ such that $a_{\emptyset}^{*} \tilde{\mathcal{L}}_{\emptyset}=b_{\emptyset}^{*} \mathcal{L}$. Note that the isomorphism class of $\tilde{\mathcal{L}}_{\emptyset}$ is independent of the choice of $d$. Assume in addition that:

(iii) $\left\{w \in W: \underline{D}(w)=w, \underline{w}^{*} \mathcal{L} \cong \mathcal{L}\right\}=\{1\}$.

We show:

(b) $p_{\emptyset !} \tilde{\mathcal{L}}_{\emptyset}$ is an irreducible intersection cohomology complex on $D$.

We identify $Z_{\emptyset}$ with the variety $X=\left\{\left(g, x B^{*}\right) \in G \times G^{0} / B^{*}: x^{-1} g x \in N B^{*}\right\}$ (as in [L3, (I), 5.4], with $\left.P=B^{*}, L=T, S=\tilde{T}_{D}\right)$ by $\left(g, x B^{*}\right) \leftrightarrow\left(x B^{*} x^{-1}, g\right)$. Then $\tilde{\mathcal{L}}_{\emptyset}$ becomes the local system $\overline{\mathcal{E}}$ on $X$ defined as in [L3, (I), 5.6], in terms of the local system $\mathcal{E}=j^{*} \mathcal{L}$ on $\tilde{T}_{D}$, where $j: \tilde{T}_{D} \rightarrow T$ is $y \mapsto d^{-1} y$. (Note that $\mathcal{E}$ is equivariant for the conjugation action of $T$ on $\tilde{T}_{D}$.) In our case we have $\overline{\mathcal{E}}=I C(X, \overline{\mathcal{E}})$ since $X$ is smooth. Hence from [L3, I, 5.7], we see that $p_{\emptyset !} \overline{\mathcal{E}}$ is an intersection cohomology complex on $D$ corresponding to a semisimple local system on an open dense subset 
of $D$ which, by the results in [L3, II, 7.10], is irreducible if and only if the following condition is satisfied: if $w \in W, x \in[w]$ satisfy $\operatorname{Ad}(x)\left(\tilde{T}_{D}\right)=\tilde{T}_{D}$ and $\operatorname{Ad}(x)^{*} \mathcal{E} \cong \mathcal{E}$, then $w=1$. This is clearly equivalent to condition (iii). This proves (b).

From (b) and the definitions we see that $p_{\emptyset !} \tilde{\mathcal{L}}_{\emptyset}[\operatorname{dim} D]$ is a character sheaf on $D$ in the sense of [L3, VI]. A character sheaf on $D$ of this form is said to be generic. We can state the following result.

Theorem 1.2. Let $A$ be a generic character sheaf on $D$ such that $F^{*} A \cong A$, where $F: D \rightarrow D$ is the restriction of $F: G \rightarrow G$. Let $\psi: F^{*} A \rightarrow A$ be an isomorphism. Define $\chi_{\psi}: D^{F} \rightarrow \overline{\mathbf{Q}}_{l}$ by $g \mapsto \sum_{i \in \mathbf{Z}}(-1)^{i} \operatorname{tr}\left(\psi, \mathcal{H}_{g}^{i}(A)\right)$, where $\mathcal{H}^{i}$ is the $i$-th cohomology sheaf and $\mathcal{H}_{g}^{i}$ is its stalk at $g$. There exists a $G^{F}$-module $V$ and a scalar $\lambda \in \overline{\mathbf{Q}}_{l}^{*}$ such that $\chi_{\psi}(g)=\lambda \operatorname{tr}(g, V)$ for all $g \in D^{F}$.

The proof is given in $\S 3$. We now make some preliminary observations. In the setup of 1.1 we have $A=p_{\emptyset !} \tilde{\mathcal{L}}_{\emptyset}[\operatorname{dim} D]$, where $\mathcal{L}$ satisfies $1.1(\mathrm{i}),(\mathrm{ii})$,(iii) and $F^{*}\left(p_{\emptyset !} \tilde{\mathcal{L}}_{\emptyset}\right) \cong p_{\emptyset !} \tilde{\mathcal{L}}_{\emptyset}$. Hence we have $p_{\emptyset !} \widetilde{F}^{*} \mathcal{L}_{\emptyset} \cong p_{\emptyset !} \tilde{\mathcal{L}}_{\emptyset}$. By a computation in [L3, IV, 21.18], we deduce that there exists $w^{\prime} \in W$ such that $\underline{D}\left(w^{\prime}\right)=w^{\prime}, \underline{w}^{\prime *} F^{*} \mathcal{L} \cong \mathcal{L}$. Setting $w=F\left(w^{\prime}\right)$ we see that

(a) $\underline{D}(w)=w, F^{*} \underline{w}^{*} \mathcal{L} \cong \mathcal{L}$.

1.3. Let $\mathbf{w}=\left(w_{1}, w_{2}, \ldots, w_{r}\right)$ be a sequence in $W$. Let $l_{\mathbf{w}}=l\left(w_{1}\right)+l\left(w_{2}\right)+\cdots+$ $l\left(w_{r}\right)$. Let

$Z_{\mathbf{w}}=\left\{\left(B_{0}, B_{1}, \ldots, B_{r}, g\right) \in \mathcal{B}^{r+1} \times D ; g B_{0} g^{-1}=B_{r},\left(B_{i-1}, B_{i}\right) \in \mathcal{O}_{w_{i}}(i \in[1, r])\right\}$.

This agrees with the definition in 1.1 when $r=0$, that is, $\mathbf{w}=\emptyset$. Let $d \in \tilde{T}_{D}$. We define $\dot{Z}_{\mathbf{w}, d}$ as in 1.1 when $r=0$ and by

$$
\begin{aligned}
\dot{Z}_{\mathbf{w}, d}=\{ & \left(h_{0} U^{*}, h_{1} B^{*}, \ldots, h_{r-1} B^{*}, h_{r} U^{*}, g\right) \in \\
\left(G^{0} / U^{*}\right) \times & \left(G^{0} / B^{*}\right) \times \ldots \times\left(G^{0} / B^{*}\right) \times\left(G^{0} / U^{*}\right) \times D ; \\
& \left.k\left(h_{i-1}^{-1} h_{i}\right) \in\left[w_{i}\right](i \in[1, r]), h_{r}^{-1} g h_{0} d^{-1} \in U^{*}\right\}
\end{aligned}
$$

when $r \geq 1$. Define $a_{\mathbf{w}}: \dot{Z}_{\mathbf{w}, d} \rightarrow Z_{\mathbf{w}}$ as in 1.1 when $r=0$ and by

$$
\begin{aligned}
& \left(h_{0} U^{*}, h_{1} B^{*}, \ldots, h_{r-1} B^{*}, h_{r} U^{*}, g\right) \mapsto \\
& \left(h_{0} B^{*} h_{0}^{-1}, h_{1} B^{*} h_{1}^{-1}, \ldots, h_{r-1} B^{*} h_{r-1}, h_{r} B^{*} h_{r}^{-1}, g\right)
\end{aligned}
$$

when $r \geq 1$. Note that $a_{\mathbf{w}}$ is a principal $T$-bundle where $T$ acts (freely) on $\dot{Z}_{\mathbf{w}, d}$ as in 1.1 when $r=0$ and by

$$
\begin{aligned}
& t_{0}:\left(h_{0} U^{*}, h_{1} B^{*}, \ldots, h_{r-1} B^{*}, h_{r} U^{*}, g\right) \mapsto \\
& \left(h_{0} t_{0}^{-1} U^{*}, h_{1} B^{*}, \ldots, h_{r-1} B^{*}, h_{r} d t_{0}^{-1} d^{-1} U^{*}, g\right)
\end{aligned}
$$

when $r \geq 1$. Define $p_{\mathbf{w}}: Z_{\mathbf{w}} \rightarrow D$ by $\left(B_{0}, B_{1}, \ldots, B_{r}, g\right) \mapsto g$.

In the remainder of this subsection we assume that $w_{1} w_{2} \ldots w_{r}=1$; this holds automatically when $r=0$. We define $b_{\mathbf{w}}: \dot{Z}_{\mathbf{w}, d} \rightarrow T$ as in 1.1 when $r=0$ and by

$$
\left(h_{0} U^{*}, h_{1} B^{*}, \ldots, h_{r-1} B^{*}, h_{r} U^{*}, g\right) \mapsto k\left(h_{0}^{-1} h_{1}\right) k\left(h_{1}^{-1} h_{2}\right) \ldots k\left(h_{r-1}^{-1} h_{r}\right)
$$

when $r \geq 1$. Note that $b_{\mathbf{w}}$ commutes with the $T$-actions where $T$ acts on $T$ as in 1.1(a).

Let $\mathcal{L}$ be a local system of rank 1 on $T$ such that 1.1(i),(ii) hold. As in 1.1, $\mathcal{L}$ is equivariant for the $T$-action 1.1(a) on $T$. Hence $b_{\mathbf{w}}^{*} \mathcal{L}$ is a $T$-equivariant local system 
on $\dot{Z}_{\mathbf{w}, d}$. Since $a_{\mathbf{w}}$ is a principal $T$-bundle, there is a well-defined local system $\tilde{\mathcal{L}}_{\mathbf{w}}$ on $Z_{\mathbf{w}}$ such that $a_{\mathbf{w}}^{*} \tilde{\mathcal{L}}_{\mathbf{w}}=b_{\mathbf{w}}^{*} \mathcal{L}$.

Lemma 1.4. Assume that $w_{1} w_{2} \ldots w_{r}=1$ and that $\mathcal{L}$ (as in section 1.3 ) satisfies

(i) $\check{\alpha}^{*} \mathcal{L} \neq \overline{\mathbf{Q}}_{l}$ for any coroot $\check{\alpha}: \mathbf{k}^{*} \rightarrow T$.

Then $p_{\mathbf{w} !} \tilde{\mathcal{L}}_{\mathbf{w}}\left[l_{\mathbf{w}}\right]\left(l_{\mathbf{w}} / 2\right) \cong p_{\emptyset !} \tilde{\mathcal{L}}_{\emptyset}$. (Note that $l_{\mathbf{w}}$ is even.)

Assume first that for some $i \in[1, r]$ we have $w_{i}=w_{i}^{\prime} w_{i}^{\prime \prime}$ where $w_{i}^{\prime}, w_{i}^{\prime \prime}$ in $W$ satisfy $l\left(w_{i}^{\prime} w_{i}^{\prime \prime}\right)=l\left(w_{i}^{\prime}\right)+l\left(w_{i}^{\prime \prime}\right)$. Let

$$
\mathbf{w}^{\prime}=\left(w_{1}, w_{2}, \ldots, w_{i-1}, w_{i}^{\prime}, w_{i}^{\prime \prime}, w_{i+1}, \ldots, w_{n}\right) .
$$

The map $\left(B_{0}, B_{1}, \ldots, B_{r+1}, g\right) \mapsto\left(B_{0}, B_{1}, B_{i-1}, B_{i+1}, \ldots, B_{r+1}, g\right)$ defines an isomorphism $Z_{\mathbf{w}^{\prime}} \rightarrow Z_{\mathbf{w}}$ compatible with the maps $p_{\mathbf{w}^{\prime}}, p_{\mathbf{w}}$ and with the local systems $\tilde{\mathcal{L}}_{\mathbf{w}^{\prime}}, \tilde{\mathcal{L}}_{\mathbf{w}}$. Since $l_{\mathbf{w}^{\prime}}=l_{\mathbf{w}}$ we have

(a) $p_{\mathbf{w} !} \tilde{\mathcal{L}}_{\mathbf{w}}\left[l_{\mathbf{w}}\right]\left(l_{\mathbf{w}} / 2\right) \cong p_{\mathbf{w}^{\prime} !} \tilde{\mathcal{L}}_{\mathbf{w}^{\prime}}\left[l_{\mathbf{w}^{\prime}}\right]\left(l_{\mathbf{w}^{\prime}} / 2\right)$.

Using (a) repeatedly we can assume that $l\left(w_{i}\right)=1$ for all $i \in[1, r]$. We will prove the result in this case by induction on $r$. Note that $r$ is even. When $r=0$ the result is obvious. We now assume that $r \geq 2$. Since $w_{1} w_{2} \ldots w_{r}=1$, we can find $j \in[1, r-1]$ such that $l\left(w_{1} w_{2} \ldots w_{j}\right)=j, l\left(w_{1} w_{2} \ldots w_{j+1}\right)=j-1$. We can find a sequence $\mathbf{w}^{\prime}=\left(w_{1}^{\prime}, w_{2}^{\prime}, \ldots, w_{r}^{\prime}\right)$ in $W$ such that $l\left(w_{i}^{\prime}\right)=1$ for all $i \in[1, r]$, $w_{1}^{\prime} w_{2}^{\prime} \ldots w_{j}^{\prime}=w_{1} w_{2} \ldots w_{j}, w_{j}^{\prime}=w_{j+1}^{\prime}, w_{i}^{\prime}=w_{i}$ for $i \in[j+1, r]$. Let

$$
\mathbf{u}=\left(w_{1} w_{2} \ldots w_{j}, w_{j+1}, \ldots, w_{r}\right)=\left(w_{1}^{\prime} w_{2}^{\prime} \ldots w_{j}^{\prime}, w_{j+1}^{\prime}, \ldots, w_{r}^{\prime}\right) .
$$

Using (a) repeatedly we see that

$$
p_{\mathbf{w} !} \mid \tilde{\mathcal{L}}_{\mathbf{w}}\left[l_{\mathbf{w}}\right]\left(l_{\mathbf{w}} / 2\right) \cong p_{\mathbf{u} !} \tilde{\mathcal{L}}_{\mathbf{u}}\left[l_{\mathbf{u}}\right]\left(l_{\mathbf{u}} / 2\right) \cong p_{\mathbf{w}^{\prime} !} \tilde{\mathcal{L}}_{\mathbf{w}^{\prime}}\left[l_{\mathbf{w}^{\prime}}\right]\left(l_{\mathbf{w}^{\prime}} / 2\right) .
$$

Replacing $\mathbf{w}$ by $\mathbf{w}^{\prime}$ we see that we may assume in addition that $w_{j}=w_{j+1}$ for some $j \in[1, r-1]$. We have a partition $Z_{\mathbf{w}}=Z_{\mathbf{w}}^{\prime} \cup Z_{\mathbf{w}}^{\prime \prime}$ where $Z_{\mathbf{w}}^{\prime}\left(\operatorname{resp} . Z_{\mathbf{w}}^{\prime \prime}\right)$ is defined by the condition $B_{j-1}=B_{j+1}$ (resp. $B_{j-1} \neq B_{j+1}$ ). Let $\mathbf{w}^{\prime}=$ $\left(w_{1}, w, \ldots, w_{j-1}, w_{j+2}, \ldots, w_{r}\right), \mathbf{w}^{\prime \prime}=\left(w_{1}, w, \ldots, w_{j-1}, w_{j+1}, \ldots, w_{r}\right)$. Define $c:$ $Z_{\mathbf{w}}^{\prime} \rightarrow Z_{\mathbf{w}^{\prime}}$ by

$$
\left(B_{0}, B_{1}, \ldots, B_{r}, g\right) \mapsto\left(B_{0}, B_{1}, \ldots, B_{j-1}, B_{j+2}, \ldots, B_{r}, g\right) .
$$

This is an affine line bundle and $\left.\tilde{\mathcal{L}}_{\mathbf{w}}\right|_{Z_{\mathbf{w}}^{\prime}}=c^{*} \tilde{\mathcal{L}}_{\mathbf{w}^{\prime}}$. Let $p_{\mathbf{w}}^{\prime}$ be the restriction of $p_{\mathbf{w}}$ to $Z_{\mathbf{w}}^{\prime}$. We have $p_{\mathbf{w}}^{\prime}=p_{\mathbf{w}^{\prime}} c$. Since the induction hypothesis applies to $\mathbf{w}^{\prime}$ we have

$$
\begin{aligned}
& p_{\mathbf{w} !}^{\prime}\left(\left.\tilde{\mathcal{L}}_{\mathbf{w}}\right|_{Z_{\mathbf{w}}^{\prime}}\right)\left[l_{\mathbf{w}}\right]\left(l_{\mathbf{w}} / 2\right)=p_{\mathbf{w}^{\prime} !} c ! c^{*} \tilde{\mathcal{L}}_{\mathbf{w}^{\prime}}\left[l_{\mathbf{w}}\right]\left(l_{\mathbf{w}} / 2\right) \\
& =p_{\mathbf{w}^{\prime} !} \tilde{\mathcal{L}}_{\mathbf{w}^{\prime}}[-2](-1)\left[l_{\mathbf{w}}\right]\left(l_{\mathbf{w}} / 2\right)=p_{\mathbf{w}^{\prime} !} \tilde{\mathcal{L}}_{\mathbf{w}^{\prime}}\left[l_{\mathbf{w}^{\prime}}\right]\left(l_{\mathbf{w}^{\prime}} / 2\right)=p_{\emptyset !} \tilde{\mathcal{L}}_{\emptyset} .
\end{aligned}
$$

Define $e: Z_{\mathbf{w}}^{\prime \prime} \rightarrow Z_{\mathbf{w}^{\prime \prime}}$ by

$$
\left(B_{0}, B_{1}, \ldots, B_{r}, g\right) \mapsto\left(B_{0}, B_{1}, \ldots, B_{j-1}, B_{j+1}, \ldots, B_{r}, g\right) .
$$

Let $p_{\mathbf{w}}^{\prime \prime}$ be the restriction of $p_{\mathbf{w}}$ to $Z_{\mathbf{w}}^{\prime \prime}$. We have $p_{\mathbf{w}}^{\prime \prime}=p_{\mathbf{w}^{\prime \prime}} e$. We show that $p_{\mathbf{w} !}^{\prime \prime}\left(\left.\tilde{\mathcal{L}}_{\mathbf{w}}\right|_{Z_{\mathbf{w}}^{\prime \prime}}\right)=0$. It is enough to show that

$$
p_{\mathbf{w}^{\prime \prime} !} e_{!}\left(\left.\tilde{\mathcal{L}}_{\mathbf{w}}\right|_{Z_{\mathbf{w}}^{\prime \prime}}\right)=0 .
$$

Hence it is enough to show that $e_{!}\left(\left.\tilde{\mathcal{L}}_{\mathbf{w}}\right|_{Z_{\mathbf{w}}^{\prime \prime}}\right)=0$. It is also enough to show that, if $E$ is a fibre of $e$, then $H_{c}^{i}\left(E,\left.\tilde{\mathcal{L}}_{\mathrm{w}}\right|_{E}\right)=0$ for any $i$. As in the proof of [L3, VI, 28.10], we may identify $E=\mathbf{k}^{*}$ in such a way that $\left.\tilde{\mathcal{L}}_{\mathbf{w}}\right|_{E}$ becomes $\check{\alpha}^{*}(\mathcal{L})$ for some coroot $\check{\alpha}: \mathbf{k}^{*} \rightarrow T$. We then use that $H_{c}^{i}\left(\mathbf{k}^{*}, \check{\alpha}^{*} \mathcal{L}\right)=0$, which follows from $\check{\alpha}^{*} \mathcal{L} \neq \overline{\mathbf{Q}}_{l}$. 
Using (c) and the exact triangle

$$
\left(p_{\mathbf{w}^{\prime \prime} !} e_{!}\left(\left.\tilde{\mathcal{L}}_{\mathbf{w}}\right|_{Z_{\mathbf{w}}^{\prime \prime}}\right), p_{\mathbf{w} !} \tilde{\mathcal{L}}_{\mathbf{w}}, p_{\mathbf{w} !}^{\prime}\left(\tilde{\mathcal{L}}_{\mathbf{w}} \mid Z_{\mathbf{w}}^{\prime}\right)\right)
$$

we see that

$$
\left.p_{\mathbf{w} !} \tilde{\mathcal{L}}_{\mathbf{w}}\left[l_{\mathbf{w}}\right]\left(l_{\mathbf{w}} / 2\right)=p_{\mathbf{w} !}^{\prime}\left(\left.\tilde{\mathcal{L}}_{\mathbf{w}}\right|_{Z_{\mathbf{w}}^{\prime}}\right)\left[l_{\mathbf{w}}\right]\right)\left(l_{\mathbf{w}} / 2\right)=p_{\emptyset !} \tilde{\mathcal{L}}_{\emptyset}
$$

(the last equality follows from (b)). The lemma is proved.

Lemma 1.5. Assume that $\mathcal{L}$ (as in section 1.3) satisfies 1.1(iii). Then $\mathcal{L}$ satisfies Lemma 1.4(i).

Let $R_{\mathcal{L}}$ be the set of roots $\alpha: T \rightarrow \mathbf{k}^{*}$ such that the corresponding coroot $\check{\alpha}$ satisfies $\check{\alpha}^{*} \mathcal{L} \cong \overline{\mathbf{Q}}_{l}$. Let $W_{\mathcal{L}}$ be the subgroup of $W$ generated by the reflections with respect to the various $\alpha \in R_{\mathcal{L}}$. Since $\underline{D}^{*} \mathcal{L} \cong \mathcal{L}$ we have $\underline{D}\left(W_{\mathcal{L}}\right)=W_{\mathcal{L}}$. Assume that Lemma 1.4(i) does not hold. Then $R_{\mathcal{L}} \neq \emptyset$ and $W_{\mathcal{L}} \neq\{1\}$. By [DL, 5.17] the fixed point set of $\underline{D}: W_{\mathcal{L}} \rightarrow W_{\mathcal{L}}$ is $\neq\{1\}$. Let $w \in W_{\mathcal{L}}-\{1\}$ be such that $\underline{D}(d) w=w$. Since $w \in W_{\mathcal{L}}$ we have $\underline{w}^{*} \mathcal{L} \cong \mathcal{L}$ (see [L3, VI, 28.3(b)]. Thus 1.1(iii) does not hold. The lemma is proved.

\section{Constructing Representations of $G^{F}$}

2.1. In this section we construct some representations of $G^{F}$ using the method of DL. See [M], DM] for other results in this direction.

Let $\mathcal{L}$ be a local system of rank 1 on $T$ such that 1.1(i) holds. For any $t \in T$ let $\mathcal{L}_{t}$ be the stalk of $\mathcal{L}$ at $t$. Assume that we are given $w \in W$ and $x \in[w]$ such that

(i) $F_{x}^{*} \mathcal{L} \cong \mathcal{L}$

$\left(F_{x}: T \rightarrow T\right.$ as in 1.1). Let $\phi: F_{x}^{*} \mathcal{L} \rightarrow \mathcal{L}$ be the unique isomorphism of local systems on $T$ which induces the identity map on $\mathcal{L}_{1}$. For $t \in T, \phi$ induces an isomorphism $\mathcal{L}_{F_{x}(t)} \stackrel{\sim}{\rightarrow} \mathcal{L}_{t}$. When $t \in T^{F_{x}}$ this is an automorphism of the 1dimensional vector space $\mathcal{L}_{t}$ given by multiplication by $\theta(t) \in \overline{\mathbf{Q}}_{l}^{*}$. It is well known that $t \mapsto \theta(t)$ is a group homomorphism $T^{F_{x}} \rightarrow \overline{\mathbf{Q}}_{l}^{*}$.

Following [DL] we define

$$
Y=\left\{h U^{*} \in G^{0} / U^{*} ; h^{-1} F(h) \in U^{*} x U^{*}\right\} .
$$

For $(g, t) \in G^{0 F} \times T^{F_{x}}$ we define $e_{g, t}: Y \rightarrow Y$ by $h U^{*} \mapsto g h t^{-1} U^{*}$. Note that $(g, t) \mapsto e_{g, t}$ is an action of $G^{0 F} \times T^{F_{x}}$ on $Y$. Hence $G^{0 F} \times T^{F_{x}}$ acts on $H_{c}^{i}(Y):=$ $H_{c}^{i}\left(Y, \overline{\mathbf{Q}}_{l}\right)$ by $(g, \tau) \mapsto e_{g^{-1}, \tau^{-1}}^{*}$. We set

$$
H_{c}^{i}(Y)_{\theta}=\left\{\xi \in H_{c}^{i}(Y): e_{1, t^{-1}}^{*} \xi=\theta(t)^{-1} \xi \text { for all } t \in T^{F_{x}}\right\} ;
$$

this is a $\left(G^{0 F} \times T^{F_{x}}\right)$-stable subspace of $H_{c}^{i}(Y)$.

For $g \in G^{0 F}$ we define $\epsilon_{g}: H_{c}^{i}(Y)_{\theta} \rightarrow H_{c}^{i}(Y)_{\theta}$ by $\epsilon_{g}(\xi)=e_{g^{-1}, 1}^{*}$. This makes $H_{c}^{i}(Y)_{\theta}$ into a $G^{0 F}$-module.

We can find an integer $r \geq 1$ such that

$$
F^{r}(x)=x, \quad x F(x) \ldots F^{r-1}(x)=1 .
$$

Indeed we first find an integer $r_{1} \geq 1$ such that $F^{r_{1}}(x)=x$ and then we find an integer $r_{2} \geq 1$ such that $\left(x F(x) \ldots F^{r_{1}-1}(x)\right)^{r_{2}}=1$. Then $r=r_{1} r_{2}$ has the required properties. Then $h U^{*} \mapsto F^{r}(h) U^{*}$ is a well-defined map $Y \rightarrow Y$ denoted again by $F^{r}$. Also,

$$
F^{r}=F_{x}^{r}: G \rightarrow G
$$


(We have $\left.F_{x}^{r}(g)=\left(x F(x) \ldots F^{r-1}(x)\right) F^{r}(g)\left(x F(x) \ldots F^{r-1}(x)\right)^{-1}=F^{r}(g).\right)$ Hence $F^{r}$ acts trivially on $T^{F_{x}}$. We see that $F^{r}: Y \rightarrow Y$ commutes with $e_{g, t}: Y \rightarrow Y$ for any $(g, t) \in G^{0 F} \times T^{F_{x}}$. Hence $\left(F^{r}\right)^{*}: H_{c}^{i}(Y) \rightarrow H_{c}^{i}(Y)$ leaves stable the subspace $H_{c}^{i}(Y)_{\theta}$. Note that:

for any $i$, all eigenvalues of $\left(F^{r}\right)^{*}: H_{c}^{i}(Y) \rightarrow H_{c}^{i}(Y)$ are of the form root of 1 times $q^{n r / 2}$ where $n \in \mathbf{Z}$.

(See [L1, 6.1(e)] and the references there.)

Replacing $r$ by an integer multiple we may therefore assume that $r$ satisfies in addition the following condition:

(a) for any $i$, all eigenvalues of $\left(F^{r}\right)^{*}: H_{c}^{i}(Y) \rightarrow H_{c}^{i}(Y)$ are of the form $q^{n r / 2}$, where $n \in \mathbf{Z}$.

2.2. We preserve the setup of section 2.1 and assume in addition that $\mathcal{L}$ satisfies Lemma 1.4(i). Let $i_{0}=2 \operatorname{dim} U^{*}-l(w)$. Note that

(a) $H_{c}^{i}(Y)_{\theta}=0$ for $i \neq i_{0}$; if $i=i_{0}$, then all eigenvalues of $\left(F^{r}\right)^{*}: H_{c}^{i}(Y)_{\theta} \rightarrow$ $H_{c}^{i}(Y)_{\theta}$ are of the form $q^{i r / 2}$.

For the first statement in (a), see [DL, 9.9] and the remarks in the proof of [L1, 8.15]. The second statement in (a) is deduced from 2.1(a) as in the proof of [L1, $6.6(\mathrm{c})]$.

2.3. We preserve the setup of section 2.1 and assume in addition that $\mathcal{L}$ satisfies 1.1(ii) and that $w \in W$ satisfies $\underline{D}(w)=w$. From the definitions we see that $\underline{D}: T \rightarrow T$ commutes with $F_{x}: T \rightarrow T$; hence $\underline{D}$ restricts to an automorphism of $T^{F_{x}}$ and

(a) $\theta(\underline{D}(t))=\theta(t)$ for any $t \in T^{F_{x}}$.

We show:

(b) there exists a homomorphism $\tilde{\theta}: \tilde{T}^{F_{x}} \rightarrow \overline{\mathbf{Q}}_{l}^{*}$ such that $\left.\tilde{\theta}\right|_{T^{F_{x}}}=\theta$.

Let $d \in \tilde{T}_{D}^{F_{x}}$. Let $n=\left|G / G^{0}\right|=\left|\tilde{T}^{F_{x}} / T^{F_{x}}\right|$. Then $t_{0}:=d^{n} \in T^{F_{x}}$. Let $c \in \overline{\mathbf{Q}}_{l}^{*}$ be such that $c^{n}=\theta\left(t_{0}\right)$. For any $t \in T^{F_{x}}$ and $j \in \mathbf{Z}$ we set $\tilde{\theta}\left(d^{j} t\right)=c^{j} \theta(t)$. This is well defined: if $d^{j} t=d^{j^{\prime}} t^{\prime}$ with $j, j^{\prime} \in \mathbf{Z}, t, t^{\prime} \in T^{F_{x}}$, then $j^{\prime}=j+n j_{0}$, $j_{0} \in \mathbf{Z}$ and $t^{\prime}=t_{0}^{j_{0}} t$ so that $\theta\left(t^{\prime}\right)=c^{n j_{0}} \theta(t)$ and $c^{j} \theta(t)=c^{j^{\prime}} \theta\left(t^{\prime}\right)$. We show that if $j, j^{\prime} \in \mathbf{Z}, t, t^{\prime} \in T^{F_{x}}$, then $\tilde{\theta}\left(d^{j} t d^{j^{\prime}} t^{\prime}\right)=\tilde{\theta}\left(d^{j} t\right) \tilde{\theta}\left(d^{j^{\prime}} t^{\prime}\right)$; that is, $c^{j+j^{\prime}} \theta\left(\underline{D}^{-j^{\prime}}(t) t^{\prime}\right)=$ $c^{j} \theta(t) c^{j^{\prime}} \theta\left(t^{\prime}\right)$. This follows from (a). This proves (b).

Let $\Gamma=\left\{(g, \tau) \in G^{F} \times \tilde{T}^{F_{x}}: g \tau^{-1} \in G^{0}\right\}$, a subgroup of $G^{F} \times \tilde{T}^{F_{x}}$. For $(g, \tau) \in \Gamma$ we define $e_{g, \tau}: Y \rightarrow Y$ by $h U^{*} \mapsto g h \tau^{-1} U^{*}$. To see that this is well defined we assume that $h \in G^{0}$ satisfies $h^{-1} F(h) \in U^{*} x U^{*}$ and $(g, \tau) \in \Gamma$; we compute

$$
\begin{aligned}
& \left(g h \tau^{-1}\right)^{-1} F\left(g h \tau^{-1}\right)=\tau h^{-1} g^{-1} g F(h) F\left(\tau^{-1}\right) \\
& =\tau h^{-1} F(h) F\left(\tau^{-1}\right) \in \tau U^{*} x U^{*} F\left(\tau^{-1}\right)=U^{*} \tau x F\left(\tau^{-1}\right) U^{*}=U^{*} x U^{*},
\end{aligned}
$$

since $\tau x F\left(\tau^{-1}\right)=x$ (that is, $\left.F_{x}(\tau)=\tau\right)$. Note that $(g, \tau) \mapsto e_{g, \tau}$ is an action of $\Gamma$ on $Y$ (extending the action of $\left.G^{0 F} \times T^{F_{x}}\right)$. Hence $\Gamma$ acts on $H_{c}^{i}(Y)$ by $(g, \tau) \mapsto e_{g^{-1}, \tau^{-1}}^{*}$. Note that $H_{c}^{i}(Y)_{\theta}$ is a $\Gamma$-stable subspace of $H_{c}^{i}(Y)$. This follows from the identity

$$
e_{g^{-1}, \tau^{-1}} e_{1, t^{-1}}=e_{1, \tau^{-1} t^{-1} \tau e^{-1}, \tau^{-1}}
$$

for $g \in G^{F}, \tau \in \tilde{T}^{F_{x}}, t \in T^{F_{x}}$, together with the identity $\theta(t)=\theta\left(\tau^{-1} t \tau\right)$, which is a consequence of (a). 
For $g \in G^{F}$ we define $\epsilon_{g}: H_{c}^{i}(Y)_{\theta} \rightarrow H_{c}^{i}(Y)_{\theta}$ by

$$
\epsilon_{g}(\xi)=\tilde{\theta}(\tau) e_{g^{-1}, \tau^{-1}}^{*}
$$

for any $\xi \in H_{c}^{i}(Y)_{\theta}$ and any $\tau \in \tilde{T}^{F_{x}}$ such that $g \tau^{-1} \in G^{0}$. Assume that $\tau^{\prime} \in \tilde{T}^{F_{x}}$ is another element such that $g \tau^{\prime-1} \in G^{0}$. Then $\tau^{\prime}=\tau t$ with $t \in T^{F_{x}}$ and

$$
\tilde{\theta}\left(\tau^{\prime}\right) e_{g^{-1}, \tau^{-1}}^{*} \xi=\tilde{\theta}(\tau) \theta(t) e_{g^{-1}, \tau^{-1}}^{*} e_{1, t^{-1}}^{*} \xi=\tilde{\theta}(\tau) e_{g^{-1}, \tau^{-1}}^{*} \xi
$$

so that $\epsilon_{g}$ is well defined. For $g, g^{\prime}$ in $G^{F}$ we choose $\tau, \tau^{\prime}$ in $\tilde{T}^{F_{x}}$ such that $g \tau^{-1} \in$ $G^{0}, g^{\prime} \tau^{\prime-1} \in G^{0}$; we have

$$
\epsilon_{g} \epsilon_{g^{\prime}} \xi=\tilde{\theta}\left(\tau^{\prime}\right) \tilde{\theta}(\tau) e_{g^{-1}, \tau^{-1}}^{*} e_{g^{\prime-1}, \tau^{\prime-1}}^{*} \xi=\tilde{\theta}\left(\tau \tau^{\prime}\right) e_{\left(g g^{\prime}\right)^{-1},\left(\tau \tau^{\prime}\right)^{-1}}^{*} \xi=\epsilon_{g g^{\prime}} \xi .
$$

We see that

$g \mapsto \epsilon_{g}$ defines a $G^{F}$-module structure on $H_{c}^{i}(Y)_{\theta}$ extending the $G^{0 F}$-module structure in section 2.1.

(Note that this extension depends on the choice of $\tilde{\theta}$.) We show:

(c) If $(g, \tau) \in \Gamma$, then $F^{r} e_{g, \tau}: Y \rightarrow Y$ is the Frobenius map of an $\mathbf{F}_{q}$-rational structure on $Y$.

Since $e_{g, t}$ is a part of a $\Gamma$-action, it has finite order. Since $F^{r}=F_{x}^{r}: G \rightarrow G$ (see section 2.1), we see that $F^{r}: Y \rightarrow Y$ commutes with $e_{g, \tau}: Y \rightarrow Y$. Hence (c) holds.

2.4. We preserve the setup of section 2.3 and assume in addition that $\mathcal{L}$ satisfies 1.3(i). Let $i_{0}=2 \operatorname{dim} U^{*}-l(w)$. Using 2.2(a), 2.3(c) and Grothendieck's trace formula we see that for $(g, d) \in \Gamma$ we have

$$
\begin{aligned}
& (-1)^{l(w)} \tilde{\theta}(d) q^{i_{0} r / 2} \operatorname{tr}\left(\epsilon_{g}, H_{c}^{i_{0}}(Y)_{\theta}\right) \\
& =\tilde{\theta}(d) \sum_{i}(-1)^{i} \operatorname{tr}\left(\left(F^{r}\right)^{*} \epsilon_{g}, H_{c}^{i}(Y)_{\theta}\right)=\sum_{i}(-1)^{i} \operatorname{tr}\left(\left(F^{r}\right)^{*} e_{g^{-1}, d^{-1}}^{*}, H_{c}^{i}(Y)_{\theta}\right) \\
& =\sum_{i}(-1)^{i}\left|T^{F_{x}}\right|^{-1} \sum_{t \in T^{F_{x}}} \operatorname{tr}\left(\left(F^{r}\right)^{*} e_{g^{-1}, d^{-1}}^{*} e_{1, t^{-1}}^{*}, H_{c}^{i}(Y)\right) \theta(t) \\
& =\left|T^{F_{x}}\right|^{-1} \sum_{t \in T^{F_{x}}} \sum_{i}(-1)^{i} \operatorname{tr}\left(\left(F^{r}\right)^{*} e_{g^{-1},(d t)^{-1}}^{*}, H_{c}^{i}(Y)\right) \theta(t) \\
& =\left|T^{F_{x}}\right|^{-1} \sum_{t \in T^{F_{x}}} \mid Y^{F^{r} e_{g^{-1},(d t)-1} \mid \theta(t)} \\
& =\left|T^{F_{x}}\right|^{-1} \sum_{t \in T^{F_{x}}}\left|\left\{h U^{*} \in\left(G^{0} / U^{*}\right): h^{-1} F(h) \in U^{*} x U^{*}, h^{-1} g^{-1} F^{r}(h) d t \in U^{*}\right\}\right| \theta(t) .
\end{aligned}
$$

\section{Proof of Theorem 1.2}

3.1. Let $A, \psi, \chi_{\psi}$ be as in Theorem 1.2. Let $\mathcal{L}, w$ be as in the end of Theorem 1.2. Let $x \in[w]$. From Theorem 1.2(a) we see that 2.1(i) holds. Let $r \geq 1$ be as in 2.1. Let

$$
\mathbf{w}=\left(w, F(w), \ldots, F^{r-1}(w)\right) .
$$

By the choice of $r$ we have $w F(w) \ldots F^{r-1}(w)=1$. Define a morphism $\tilde{F}: Z_{\mathbf{w}} \rightarrow$ $Z_{\mathbf{w}}$ by

$$
\tilde{F}\left(B_{0}, B_{1}, \ldots, B_{r}, g\right)=\left(F\left(g^{-1} B_{r-1} g\right), F\left(B_{0}\right), F\left(B_{1}\right), \ldots, F\left(B_{r-1}\right), F(g)\right) .
$$


We show:

(a) Let $g \in D^{F}$ and let $\tilde{F}_{g}: p_{\mathbf{w}}^{-1}(g) \rightarrow p_{\mathbf{w}}^{-1}(g)$ be the restriction of $\tilde{F}: Z_{\mathbf{w}} \rightarrow Z_{\mathbf{w}}$. Then $\tilde{F}_{g}$ is the Frobenius map of an $\mathbf{F}_{q}$-rational structure on $p_{\mathbf{w}}^{-1}(g)$. It is enough to note that the map $\mathcal{B}^{r+1} \rightarrow \mathcal{B}^{r+1}$ given by

$$
\left(B_{0}, B_{1}, \ldots, B_{r}\right) \mapsto\left(F\left(g^{-1} B_{r-1} g\right), F\left(B_{0}\right), F\left(B_{1}\right), \ldots, F\left(B_{r-1}\right)\right)
$$

is the composition of the map

$$
F^{\prime}:\left(B_{0}, B_{1}, \ldots, B_{r}\right) \mapsto\left(F\left(B_{0}\right), F\left(B_{1}\right), \ldots, F\left(B_{r}\right)\right)
$$

(the Frobenius map of an $\mathbf{F}_{q}$-rational structure on $\mathcal{B}^{r+1}$ ) with the automorphism

$$
\left(B_{0}, B_{1}, \ldots, B_{r}\right) \mapsto\left(g^{-1} B_{r-1} g, B_{0}, B_{1}, \ldots, B_{r-1}\right)
$$

of $\mathcal{B}^{r+1}$ which commutes with $F^{\prime}$ and has finite order (since $g$ has finite order in $G$ ).

Let $d \in \tilde{T}_{D}^{F_{x}}$. Define a morphism $\tilde{F}^{\prime}: \dot{Z}_{\mathbf{w}, d} \rightarrow \dot{Z}_{\mathbf{w}, d}$ by

$$
\tilde{F}^{\prime}\left(h_{0} U^{*}, h_{1} B^{*}, \ldots, h_{r-1} B^{*}, h_{r} U^{*}, g\right)=\left(h_{0}^{\prime} U^{*}, h_{1}^{\prime} B^{*}, \ldots, h_{r-1}^{\prime} B^{*}, h_{r}^{\prime} U^{*}, F(g)\right),
$$

where

$$
\begin{gathered}
h_{0}^{\prime}=F\left(g^{-1} h_{r-1} k\left(h_{r-1}^{-1} h_{r}\right)\right) x^{-1} d, \quad h_{r}^{\prime}=F\left(h_{r-1} k\left(h_{r-1}^{-1} h_{r}\right)\right) x^{-1}, \\
h_{i}^{\prime}=F\left(h_{i-1}\right) \text { for } i \in[1, r-1] .
\end{gathered}
$$

This is well defined since

$$
\left(F\left(h_{r-1} k\left(h_{r-1}^{-1} h_{r}\right) x^{-1}\right)^{-1} F(g) F\left(g^{-1} h_{r-1} k\left(h_{r-1}^{-1} h_{r}\right)\right) x^{-1}\right) d d^{-1}=1 .
$$

We show that the $T$-action on $\dot{Z}_{\mathbf{w}, d}$ (see section 1.3) satisfies $\tilde{F}^{\prime}\left(t_{0} \tilde{x}\right)=$ $F_{x}\left(t_{0}\right) \tilde{F}^{\prime}(\tilde{x})$ for $t_{0} \in T, \tilde{x} \in \dot{Z}_{\mathbf{w}, d}$. Let $\left(h_{i}\right)$ be as above. We must show:

$$
\begin{gathered}
F\left(g^{-1} h_{r-1} k\left(h_{r-1}^{-1} h_{r} d t_{0}^{-1} d^{-1}\right)\right) x^{-1} d=F\left(g^{-1} h_{r-1} k\left(h_{r-1}^{-1} h_{r}\right)\right) x^{-1} d x F\left(t_{0}^{-1}\right) x^{-1}, \\
F\left(h_{r-1} k\left(h_{r-1}^{-1} h_{r} d t_{0}^{-1} d^{-1}\right)\right) x^{-1}=F\left(h_{r-1} k\left(h_{r-1}^{-1} h_{r}\right) x^{-1} d x F\left(t_{0}\right)\right)^{-1} x^{-1} d^{-1},
\end{gathered}
$$

which follow from $F(d)=x^{-1} d x$. Note that

(b) $a_{\mathbf{w}} \tilde{F}^{\prime}=\tilde{F} a_{\mathbf{w}}: \dot{Z}_{\mathbf{w}, d} \rightarrow Z_{\mathbf{w}}$.

We show:

(c) $\left|a_{\mathbf{w}}^{-1}(y)^{\tilde{F}^{\prime}}\right|=\left|T^{F_{x}}\right|$ for any $y \in Z_{\mathbf{w}}^{\tilde{F}}$.

Since $a_{\mathbf{w}}^{-1}(y)$ is a homogeneous $T$-space, this follows from Lang's theorem applied to $\left(T, F_{x}\right)$.

We have

(d) $p_{\mathbf{w}} \tilde{F}=F p_{\mathbf{w}}: Z_{\mathbf{w}} \rightarrow D$.

3.2. We show:

(a) $b_{\mathbf{w}} \tilde{F}^{\prime}=F_{x} b_{\mathbf{w}}: \dot{Z}_{\mathbf{w}, d} \rightarrow T$.

Let $\left(h_{0}, h_{1}, \ldots, h_{r}, g\right) \in\left(G^{0}\right)^{r+1} \times D$ be such that

$$
\left(h_{0} U^{*}, h_{1} B^{*}, \ldots, h_{r-1} B^{*}, h_{r} U^{*}, g\right) \in \dot{Z}_{\mathbf{w}, d} .
$$

Let $\left(h_{1}^{\prime}, h_{2}^{\prime}, \ldots, h_{r}^{\prime}\right)$ be as in section 3.1. We set

$$
\begin{gathered}
\mu=k\left(h_{0}^{-1} h_{1}\right) k\left(h_{1}^{-1} h_{2}\right) \ldots k\left(h_{r-1}^{-1} h_{r}\right) \in T, \\
\mu^{\prime}=k\left(h_{0}^{-1} h_{1}\right) k\left(h_{1}^{-1} h_{2}\right) \ldots k\left(h_{r-2}^{-1} h_{r-1}\right) \in B^{*} F^{r-1}(x)^{-1} B^{*} \\
\tilde{\mu}=k\left(h_{0}^{\prime-1} h_{1}^{\prime}\right) k\left(h_{1}^{\prime-1} h_{2}^{\prime}\right) \ldots k\left(h_{r-1}^{\prime}{ }^{-1} h_{r}^{\prime}\right) \in T
\end{gathered}
$$


so that $\mu=\mu^{\prime} k\left(h_{r-1}^{-1} h_{r}\right)$ and

$$
\begin{aligned}
& \tilde{\mu}=k\left(d^{-1} x F\left(k\left(h_{r-1}^{-1} h_{r}\right)^{-1} h_{r-1}^{-1} g h_{0}\right)\right) \\
& \times k\left(F\left(h_{0}^{-1} h_{1}\right)\right) \ldots k\left(F\left(h_{r-3}^{-1} h_{r-2}\right)\right) k\left(F\left(h_{r-2}^{-1} h_{r-1} k\left(h_{r-1}^{-1} h_{r}\right)\right) x^{-1}\right) \\
& =d^{-1} x F\left(k\left(h_{r-1}^{-1} h_{r}\right)^{-1}\right) F(d) k\left(F\left(d^{-1}\right) F\left(h_{r-1}^{-1} g h_{0}\right)\right) F\left(\mu^{\prime}\right) F\left(k\left(h_{r-1}^{-1} h_{r}\right)\right) x^{-1} \\
& =d^{-1} x F(d) F(\mu) x^{-1}=x F(\mu) x^{-1}=F_{x}(\mu),
\end{aligned}
$$

as required.

3.3. Let $\phi: F_{x}^{*} \mathcal{L} \stackrel{\sim}{\rightarrow} \mathcal{L}, \theta: T^{F_{x}} \rightarrow \overline{\mathbf{Q}}_{l}^{*}$ be as in section 2.1 . We shall denote by ? the various isomorphisms induced by $\phi$ such as:
(a) $\tilde{F}^{\prime *} b_{\mathrm{w}}^{*} \mathcal{L}=b_{\mathrm{w}}^{*} F_{x}^{*} \mathcal{L} \stackrel{\sim}{\rightarrow} b_{\mathrm{w}}^{*} \mathcal{L}($ see $3.2(\mathrm{a}))$,
(b) $\tilde{F}^{* *} a_{\mathrm{w}}^{*} \tilde{\mathcal{L}}_{\mathrm{w}} \stackrel{\sim}{\rightarrow} a_{\mathrm{w}}^{*} \tilde{\mathcal{L}}_{\mathrm{w}}$ (coming from (a)),
(c) $a_{\mathbf{w}}^{*} \tilde{F}^{*} \tilde{\mathcal{L}}_{\mathbf{w}} \stackrel{\sim}{\rightarrow} a_{\mathbf{w}}^{*} \tilde{\mathcal{L}}_{\mathbf{w}}$ (see (b) and 3.1(b)),
(d) $\tilde{F}^{*} \tilde{\mathcal{L}}_{\mathrm{w}} \stackrel{\sim}{\rightarrow} \tilde{\mathcal{L}}_{\mathrm{w}}$ (coming from (c)),
(e) $p_{\mathbf{w} !} \tilde{F}^{*} \tilde{\mathcal{L}}_{\mathbf{w}} \stackrel{\sim}{\rightarrow} p_{\mathbf{w} !} \tilde{\mathcal{L}}_{\mathbf{w}}$ (coming from (d)),
(f) $F^{*} p_{\mathbf{w} !} \tilde{\mathcal{L}}_{\mathrm{w}} \stackrel{\sim}{\rightarrow} p_{\mathrm{w}} ! \tilde{\mathcal{L}}_{\mathrm{w}}($ coming from $(\mathrm{e})$ and $3.1(\mathrm{~d}))$,
(g) $F^{*}\left(p_{\mathbf{w} !} \tilde{\mathcal{L}}_{\mathbf{w}}\left[l_{\mathbf{w}}\right]\right) \stackrel{\sim}{\rightarrow} p_{\mathbf{w} !} \tilde{\mathcal{L}}_{\mathbf{w}}\left[l_{\mathbf{w}}\right]($ coming from $(\mathrm{f}))$.

3.4. For any $g \in D^{F}$ we compute

$$
\begin{aligned}
& \sum_{i}(-1)^{i} \operatorname{tr}\left(?, \mathcal{H}_{g}^{i}\left(p_{\mathbf{w} !} \tilde{\mathcal{L}}_{\mathbf{w}}\right)\right)=\sum_{i}(-1)^{i} \operatorname{tr}\left(?, H_{c}^{i}\left(p_{\mathbf{w}}^{-1}(g), \tilde{\mathcal{L}}_{\mathbf{w}}\right)\right) \\
& =\sum_{y \in p_{\mathbf{w}}^{-1}(g) ; \tilde{F}(y)=y} \operatorname{tr}\left(?,\left(\tilde{\mathcal{L}}_{\mathbf{w}}\right)_{y}\right)
\end{aligned}
$$

where $\mathcal{H}^{i}$ is the $i$-th cohomology sheaf. (The last two sums are equal by the Grothendieck trace formula applied in the context of 3.1(a).) Using 3.1(c) we see that the last sum equals

$$
\begin{aligned}
& \left|T^{F_{x}}\right|^{-1} \sum_{\tilde{y} \in a_{\mathbf{w}}^{-1}\left(p_{\mathbf{w}}^{-1}(g)\right)^{\tilde{F}^{\prime}}} \operatorname{tr}\left(?,\left(a_{\mathbf{w}}^{*} \tilde{\mathcal{L}}_{\mathbf{w}}\right) \tilde{y}\right)=\left|T^{F_{x}}\right|^{-1} \sum_{\tilde{y} \in a_{\mathbf{w}}^{-1}\left(p_{\mathbf{w}}^{-1}(g)\right)^{\tilde{F}^{\prime}}} \operatorname{tr}\left(?,\left(b_{\mathbf{w}}^{*} \mathcal{L}_{\mathbf{w}}\right) \tilde{y}\right) \\
& =\left|T^{F_{x}}\right|^{-1} \sum_{\tilde{y} \in a_{\mathbf{w}}^{-1}\left(p_{\mathbf{w}}^{-1}(g)\right)^{\tilde{F}^{\prime}}} \operatorname{tr}\left(?,\left(\mathcal{L}_{\mathbf{w}}\right)_{b_{\mathbf{w}}(\tilde{y})}\right) .
\end{aligned}
$$

Now $a_{\mathbf{w}}^{-1}\left(p_{\mathbf{w}}^{-1}(g)\right)^{\tilde{F}^{\prime}}$ can be identified with the set of all

$$
\left(h_{0} U^{*}, h_{1} B^{*}, \ldots, h_{r-1} B^{*}, h_{r} U^{*}\right) \in\left(G^{0} / U^{*}\right) \times\left(G^{0} / B^{*}\right) \times \ldots \times\left(G^{0} / B^{*}\right) \times\left(G^{0} / U^{*}\right)
$$

such that

(a) $k\left(h_{i-1}^{-1} h_{i}\right) \in F^{i-1}(x) T$ for $i \in[1, r]$

(b) $h_{r}^{-1} g h_{0} d^{-1} \in U^{*}$,

(c) $h_{0} U^{*}=F\left(g^{-1} h_{r-1} k\left(h_{r-1}^{-1} h_{r}\right)\right) x^{-1} d U^{*}$,

(d) $h_{i} B^{*}=F\left(h_{i-1}\right) B^{*}$ for $i \in[1, r-1]$.

(We then have automatically $h_{r} U^{*}=F\left(h_{r-1} k\left(h_{r-1}^{-1} h_{r}\right) x^{-1} U^{*}\right.$.) If $h_{0} U^{*}$ is given, then (d) determines successively $h_{2} B^{*}, \ldots h_{r-1} B^{*}$ in a unique way and (b) determines $h_{r} U^{*}$ in a unique way. We see that the equations (a)-(d) are equivalent to 
the following equations for $h_{0} U^{*}$ :

$$
\begin{gathered}
h_{0}^{-1} F\left(h_{0}\right) \in B^{*} x B^{*}, \quad F^{r-1}\left(h_{0}\right)^{-1} g h_{0} d^{-1} \in B^{*} F^{r-1}(x) B^{*}, \\
F^{r}\left(h_{0}\right)^{-1} g h_{0} d^{-1} U^{*}=k\left(F^{r}\left(h_{0}\right)^{-1} g F\left(h_{0}\right) F\left(d^{-1}\right)\right) x^{-1} U^{*}
\end{gathered}
$$

(if $r \geq 2$ ) and

$$
h_{0}^{-1} g h_{0} d^{-1} \in B^{*} x B^{*}, \quad F\left(h_{0}\right)^{-1} g h_{0} d^{-1} U^{*}=k\left(F\left(h_{0}\right)^{-1} g F\left(h_{0}\right) F\left(d^{-1}\right)\right) x^{-1} U^{*}
$$

(if $r=1$ ). In both cases these equations are equivalent to

$$
h_{0}^{-1} F\left(h_{0}\right) \in U^{*} t x F(t)^{-1} U^{*}, \quad F^{r}\left(h_{0}\right)^{-1} g h_{0} d^{-1} \in F^{r}(t) U^{*}
$$

for some $t \in T$. We then have $F^{r-1}\left(h_{0}\right)^{-1} g h_{0} d^{-1} \in U^{*} F^{r-1}(t) F^{r-1}(x) U^{*}$. For $h_{0} U^{*}, t$ as in (e) we compute

$$
\begin{aligned}
& k\left(h_{0}^{-1} F\left(h_{0}\right)\right) k\left(F\left(h_{0}\right)^{-1} F^{2}\left(h_{0}\right)\right) \ldots k\left(F^{r-2}\left(h_{0}\right)^{-1} F^{r-1}\left(h_{0}\right)\right) k\left(F^{r-1}\left(h_{0}\right)^{-1} g h_{0} d^{-1}\right) \\
& =\left(t x F(t)^{-1}\right)\left(F(t) F(x) F^{2}\left(t^{-1}\right)\right) \ldots\left(F^{r-2}(t) F^{r-2}(x) F^{r-1}(t)^{-1}\right)\left(F^{r-1}(t) F^{r-1}(x)\right) \\
& =t x F(x) \ldots F^{r-1}(x)=t .
\end{aligned}
$$

By 3.2(a) the result of the last computation is necessarily in $T^{F_{x}}$. Thus $F_{x}(t)=t$. Hence $F^{r}(t)=t$ and the equations (e) become

$$
h_{0}^{-1} F\left(h_{0}\right) \in U^{*} x U^{*}, \quad F^{r}\left(h_{0}\right)^{-1} g h_{0} d^{-1} \in T^{F_{x}} U^{*} .
$$

We see that

$$
\sum_{i}(-1)^{i} \operatorname{tr}\left(?, \mathcal{H}_{g}^{i}\left(p_{\mathbf{w} !} \tilde{\mathcal{L}}_{\mathbf{w}}\right)\right)=\left|T^{F_{x}}\right|^{-1} \sum_{t \in T^{F_{x}}} a_{t}=\left|T^{F_{x}}\right|^{-1} \sum_{t^{\prime} \in T^{F_{x}}} a_{t^{\prime}}^{\prime}
$$

where

$$
\begin{gathered}
a_{t}=\left|\left\{h U^{*} \in\left(G^{0} / U^{*}\right): h^{-1} F(h) \in U^{*} x U^{*}, d h^{-1} g^{-1} F^{r}(h) t \in U^{*}\right\}\right| \theta(t), \\
a_{t^{\prime}}^{\prime}=\left|\left\{h U^{*} \in\left(G^{0} / U^{*}\right): h^{-1} F(h) \in U^{*} x U^{*}, h^{-1} g^{-1} F^{r}(h) d t^{\prime} \in U^{*}\right\}\right| \theta\left(d t^{\prime} d^{-1}\right) .
\end{gathered}
$$

Comparing with the last formula in section 2.4 and using $\theta\left(d t^{\prime} d^{-1}\right)=\theta\left(t^{\prime}\right)$ for $t^{\prime} \in T^{F_{x}}$ we obtain (with $i_{0}$ as in section 2.4):

$$
\sum_{i}(-1)^{i} \operatorname{tr}\left(?, \mathcal{H}_{g}^{i}\left(p_{\mathbf{w} !} \tilde{\mathcal{L}}_{\mathbf{w}}\right)\right)=(-1)^{l(w)} \tilde{\theta}(d) q^{i_{0} r / 2} \operatorname{tr}\left(\epsilon_{g}, H_{c}^{i_{0}}(Y)_{\theta}\right) .
$$

Let us choose an isomorphism $p_{\mathbf{w} !} \tilde{\mathcal{L}}_{\mathbf{w}}\left[l_{\mathbf{w}}\right] \cong p_{\emptyset !} \tilde{\mathcal{L}}_{\emptyset}$. (This exists by Lemma 1.4; note that Lemma 1.4(i) holds by Lemma 1.5.) Via this isomorphism, the isomorphism $3.3(\mathrm{~g})$ corresponds to an isomorphism $F^{*}\left(p_{\emptyset !} \tilde{\mathcal{L}}_{\emptyset}\right) \rightarrow p_{\emptyset !} \tilde{\mathcal{L}}_{\emptyset}$, that is, to an isomorphism $\psi^{\prime}: F^{*} A \stackrel{\sim}{\rightarrow} A$ so that

$$
\sum_{i}(-1)^{i} \operatorname{tr}\left(?, \mathcal{H}_{g}^{i}\left(p_{\mathbf{w} !} \tilde{\mathcal{L}}_{\mathbf{w}}\right)\right)=\sum_{i}(-1)^{i} \operatorname{tr}\left(\psi^{\prime}, \mathcal{H}_{g}^{i}(A)\right)
$$

for any $g \in D^{F}$. (We use that $l_{\mathbf{w}}$ is even.) Since $A$ is irreducible, we must have $\psi=\lambda^{\prime} \psi^{\prime}$ for some $\lambda^{\prime} \in \overline{\mathbf{Q}}_{l}^{*}$. It follows that

$$
\sum_{i \in \mathbf{Z}}(-1)^{i} \operatorname{tr}\left(\psi, \mathcal{H}_{g}^{i}(A)\right)=\lambda^{\prime}(-1)^{l(w)} \tilde{\theta}(d) q^{i_{0} r / 2} \operatorname{tr}\left(\epsilon_{g}, H_{c}^{i_{0}}(Y)_{\theta}\right)
$$

for any $g \in D^{F}$. Thus Theorem 1.2 holds with $V$ being the $G^{F}$-module $H_{c}^{i_{0}}(Y)_{\theta}$, which is irreducible (even as a $G^{0 F}$-module) if $G^{0}$ has connected centre, but is not necessarily irreducible in general. 


\section{REFERENCES}

[DL] P. Deligne and G. Lusztig, Representations of reductive groups over finite fields, Ann. Math. 103 (1976), 103-161. MR0393266 (52:14076)

[DM] F. Digne and J. Michel, Groupes réductifs non connexes, Ann. Sci. École Norm. Sup. 27 (1994), 345-406. MR.1272294 (95f:20068)

[L1] G. Lusztig, Green functions and character sheaves, Ann. Math. 131 (1990), 355-408. MR:1043271 (91c:20054)

[L2] G. Lusztig, Remarks on computing irreducible characters, J. Amer. Math. Soc. 5 (1992), 971-986. MR.1157292 (93g:20092)

[L3] G. Lusztig, Character sheaves on disconnected groups, I, Represent. Th. (electronic) 7 (2003), 374-403; II 8 (2004), 72-124; III 8 (2004), 125-144; IV 8 (2004), 145-178; Errata, 8 (2004), 179-179; V 8 (2004), 346-376; VI 8 (2004), 377-413; VII 9 (2005), 209-266; VIII 10 (2006), 314-352; IX 10 (2006), 353-379. MR2017063 (2006d:20090a)

[M] G. Malle, Generalized Deligne-Lusztig characters, J. Algebra 159 (1993), 64-97. MR.1231204 (94i:20025)

[S] T. Shoji, Character sheaves and almost characters of reductive groups, Adv. Math. 111 (1995), 244-313; II 111 (1995), 314-354. MR1318530 (95k:20069)

[W] J.-L. Waldspurger, Le groupe $G L_{n}$ tordu sur un corps fini, Nagoya Math. J. 182 (2006), 313-379. MR2235346(2007j:20015)

Department of Mathematics, Massachusetts Institute of Technology, Cambridge, MassachusetTs 02139

E-mail address: gyuri@math.mit.edu 